\title{
Clinical Usefulness of Perioperative C-reactive Protein/ Albumin Ratio in Patients With Intrahepatic Cholangiocarcinoma: A Retrospective Single Institutional Study
}

\author{
YOSUKE NAKAO, YO-ICHI YAMASHITA, KOTA ARIMA, TATSUNORI MIYATA, RUMI ITOYAMA, \\ TOSHIHIKO YUSA, NAOKI UMEZAKI, TAKANOBU YAMAO, SHIGEKI NAKAGAWA, \\ HIROHISA OKABE, KATSUNORI IMAI, AKIRA CHIKAMOTO and HIDEO BABA \\ Department of Gastroenterological Surgery, Graduate School of Life Sciences, \\ Kumamoto University, Kumamoto, Japan
}

\begin{abstract}
Background/Aim: Prognoses of patients with cancer can be predicted on the basis of preoperative nutrition- or inflammation-based scores; however, predicting the prognostic impact of undergoing surgery remains challenging. In this study, we investigated the usefulness of the perioperative C-reactive protein/albumin (CRP/Alb) ratio in patients with intrahepatic cholangiocarcinoma (ICC). Patients and Methods: We retrospectively investigated 80 patients who had undergone curative resection of primary ICC between April 2002 and December 2017. We identified the time at which perioperative CRP/Alb ratio most influences the prognosis, and investigated the correlations among the perioperative CRP/Alb ratio, clinicopathological features and patient outcomes. Results: The only perioperative CRP/Alb ratios significantly associated with shorter overall survival $(O S)$ was a high CRP/Alb ratio on POD14. High CRP/Alb ratio on POD 14 was significantly associated with older age, male sex, and the presence of postoperative complications. Finally, a high CRP/Alb ratio at POD 14 was an independent prognostic factor for poor OS. Conclusion: CRP/Alb ratio on POD 14 may be a useful prognostic factor in patients with ICC who have undergone curative resections.
\end{abstract}

Intrahepatic cholangiocarcinoma (ICC) is the second most common primary liver cancer after hepatocellular carcinoma

Correspondence to: Yo-ichi Yamashita, MD, Ph.D., F.A.C.S, Department of Gastroenterological Surgery, Graduate School of Life Sciences, Kumamoto University, 1-1-1 Honjo, Chuo-ku, Kumamoto 860-0811, Japan. Tel: +81 963735211, Fax: +81 963714378, e-mail: y-yama@kumamoto-u.ac.jp

Key Words: Perioperative C-reactive protein/albumin ratio, intrahepatic cholangiocarcinoma, prognosis.
(HCC) (1). Although the incidence of ICC is increasing in the world, patients with ICC still have a poor prognosis, when compared to other cancers. The 5-year survival rate is only $3-31 \%$ because of frequent lymph node involvement, intrahepatic metastasis, and/or refractoriness to chemotherapy or radiation therapy (2-4). Therefore, the treatment of advanced ICC is shifting toward multidisciplinary approaches to improve patients' prognosis; however, surgical resection is currently considered the only curative treatment for ICC.

Predicting a patient's prognosis preoperatively on the basis of reliable biomarkers is very important in facilitating selection of the most appropriate treatment and postoperative follow-up strategies. Some studies have demonstrated that carbohydrate antigen 19-9 (CA19-9) is one of the most reliable postoperative prognostic markers (5). It has also been demonstrated that cancer-related inflammation is associated with tumor cell survival, proliferation, and metastasis $(6,7)$. Thus, biomarkers of cancer-related inflammation, such as preoperative neutrophil-to-lymphocyte ratio (NLR), platelet-to-lymphocyte ratio (PLR), and modified Glasgow prognostic score (mGPS) are established strong prognostic factors after curative resections of various cancers, including ICC (8-10). The mGPS is based on serum C-reactive protein (CRP) and albumin concentrations. It was recently reported that the CRP-to-albumin (CRP/Alb) ratio is associated with poor prognosis in patients with emergency medical admissions and sepsis $(11,12)$. In addition, the CRP/Alb ratio is reportedly a more important prognostic factor than mGPS in patients with HCC (13).

Hepatic resection is the only curative treatment for ICC; however, this modality is invasive and has a high treatmentrelated mortality and morbidity (14). Postoperative complications were recently reported to be related with recurrence-free survival (15). Thus, postoperative management is very important not only for postoperative quality of life, but also for prognosis. Various types of scores for predicting 
Table I. Perioperative CRP/Alb ratios and patient prognosis.

\begin{tabular}{lc}
\hline Timing of measurement & Postoperative prognosis $(p$-Value $)$ \\
\hline Preoperative $(>0.024)$ & 0.420 \\
POD $1(>0.737)$ & 0.122 \\
POD $3(>2.077)$ & 0.543 \\
POD $7(>0.732)$ & 0.054 \\
POD $14(>0.304)$ & 0.008 \\
POM $1(>0.094)$ & 0.069 \\
POM $3(>0.034)$ & 0.071 \\
\hline
\end{tabular}

POD: Postoperative day; POM: postoperative month.

whether patients can withstand surgery have been reported; however, the scores cannot predict patients' responses to undergoing radical surgery. In fact, clinical data sometimes change unexpectedly, especially when patients have undergone considerable surgical stress (16). Nevertheless, the relationship between prognostic scores based on postoperative inflammation and outcomes has not been established for ICC. Thus, we aimed to assess whether the perioperative CRP/Alb ratio influences the outcomes of patients with ICC after curative surgical resection.

\section{Patients and Methods}

Patients. Between April 2002 and December 2017, 100 patients with ICC underwent hepatic resection at our Institution. Of these 100 patients, 14 who had undergone repeated resection for ICC recurrence and six who had undergone non-curative resection were excluded; thus the final study cohort comprised 80 patients. As previously reported, surgical procedures were selected according to the size, number and site of the ICC and the patient's general and medical condition (17). CRP/Alb ratio was measured preoperatively (1-5 days before surgical resection), on postoperative days (POD) $1,3,7,14$, and on postoperative months (POM) 1 and 3. Our institution's Ethics Committee approved this study (\#1291), and informed consent was obtained from all patients in accordance with Institutional Review Board protocols.

Surgical procedures. The details of our surgical indication and technique have been reported previously $(17,18)$. We do not routinely perform lymph node (LN) dissection, only when patients are suspected of having $\mathrm{LN}$ metastases on the basis of preoperative computed tomography scan, positron emission tomography, or macroscopic findings during the procedure $(19,20)$. For example, we suspect LN metastasis if LNs are $10 \mathrm{~mm}$ or larger on preoperative imaging or a hard texture is noted during the procedure. In such cases, we mainly perform $\mathrm{LN}$ dissection at \#8 and \#12 (19). Postoperative complications are defined as Grade III or greater according to the Clavien-Dindo classification (CD) (21).

Follow-up strategies. Clinical data, such as patient background characteristics, operative results, and pathological factors, were retrospectively obtained by the medical records of the 80 study patients. All patients involved were monitored either until March

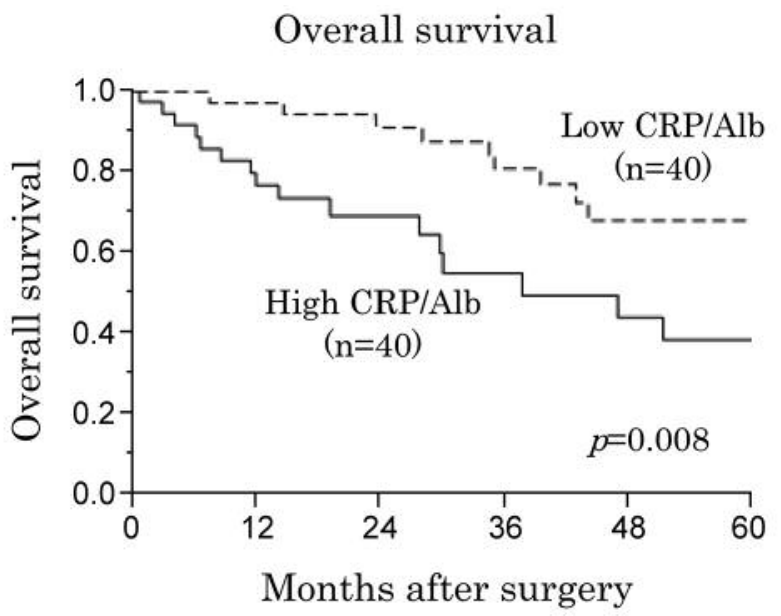

Figure 1. Overall survival rates of patients with ICC who have undergone curative resection, according to CRP/Alb ratio on POD14. The high CRP/Alb group had a significantly poorer prognosis $(p=0.008)$.

2018 or their deaths. The mean follow-up period was 37 months. The ICCs were staged in accordance with the seventh edition of the American Joint Committee on Cancer (AJCC)/International Union Against Cancer (UICC) staging manual (22). Patients were followed up at our own hospital or at affiliated hospitals; tumor markers such as carcinoembryonic antigen (CEA) and carbohydrate antigen 19-9 (CA19-9), and CT or MRI were assessed every 3 or 4 months (18).

Statistical analysis. Continuous values were expressed as median (range). Continuous and categorical variables were compared using $\chi^{2}$ tests or Student's $t$-tests, respectively. Survival analyses were performed using the Kaplan-Meier method, with comparisons by means of the log-rank test. Independent prognostic factors for poor OS were identified by multivariate Cox proportional hazard analysis and the clinicopathological factors associated with high CRP/Alb ratio were identified by stepwise logistic analysis. All cutoff points for CRP/Alb ratio are median values. The cut-off points for blood loss and operative time are also median values. All results with twotailed values of $p<0.05$ were considered to be statistically significant. All statistical analyses were performed using JMP software (Version 12; SAS Institute, Cary, NC, USA).

\section{Results}

Relationship between perioperative $C R P / A l b$ ratios and prognosis. To identify which perioperative $\mathrm{CRP} / \mathrm{Alb}$ ratio most accurately predicts patients' prognosis, CRP/Alb ratios were measured preoperatively, on POD1, POD3, POD7, POD14, POM1, and POM3. Patients were divided into high and low CRP/Alb groups according to the median value and the data subjected to Cox proportional hazard analysis for OS. The median CRP/Alb ratios were 0.024, 0.74, 2.08, $0.73,0.30,0.09$, and 0.03 preoperatively and on POD1, POD3, POD7, POD14, POM1, and POM3, respectively. The 
Table II. Patient characteristics according to CRP/Alb ratio on POD 14.

\begin{tabular}{|c|c|c|c|}
\hline \multirow{2}{*}{ Variables } & \multicolumn{2}{|c|}{$\mathrm{CRP} / \mathrm{Alb}$ at POD 14} & \multirow{2}{*}{$p$-Value } \\
\hline & $\begin{array}{c}\text { Low }(\leq 0.30) \\
n=40\end{array}$ & $\begin{array}{c}\text { High }(>0.30) \\
n=40\end{array}$ & \\
\hline Age (years) & $65.5(38-85)$ & $71(37-96)$ & 0.0829 \\
\hline Gender (male/female) & $18 / 22$ & $31 / 9$ & 0.0025 \\
\hline HBs-Ag(positive/negative) & $6 / 34$ & $2 / 38$ & 0.1281 \\
\hline $\mathrm{HCV}-\mathrm{Ab}$ (positive/negative) & $8 / 32$ & $6 / 34$ & 0.5556 \\
\hline Liver damage $(\mathrm{A} / \mathrm{B})$ & $39 / 1$ & $39 / 1$ & 0.9855 \\
\hline ICG R15 $(\%)$ & $7.8(0.8-27.6)$ & $11.2(1.9-27.3)$ & 0.1460 \\
\hline $\mathrm{CEA}(\mathrm{ng} / \mathrm{ml})$ & $2.3(0.7-36.8)$ & $2.25(0.7-2102)$ & 0.4355 \\
\hline CA19-9 (U/1) & $20.65(0.2-8223)$ & $26.85(0.1-53373)$ & 0.9539 \\
\hline Total bilirubin (mg/dl) & $0.8(0.1-1.7)$ & $0.7(0.3-1.6)$ & 0.1122 \\
\hline Albumin $(\mathrm{g} / \mathrm{dl})$ & $4.2(3.4-5.1)$ & $3.9(2.7-4.8)$ & 0.0182 \\
\hline PT $(\%)$ & $100(1.3-143)$ & $100(21-131)$ & 0.6613 \\
\hline AST (U/1) & $29(12-163)$ & $30(7-134)$ & 0.7722 \\
\hline ALT $(U / 1)$ & $25(5-285)$ & $23(7-188)$ & 0.4708 \\
\hline CRP & $0.06(0.01-0.99)$ & $0.16(0.01-2.1)$ & 0.0017 \\
\hline
\end{tabular}

ALT: Alanine transaminase; AST: aspartate transaminase; CA19-9: carbohydrate antigen 19-9; CEA: carcinoembryonic antigen; CRP: C-reactive protein; $\mathrm{HBs}-\mathrm{Ag}$ : hepatitis B surface antigen; $\mathrm{HCV}-\mathrm{Ab}$ : hepatitis $\mathrm{C}$ virus antibody; PT: prothrombin time.

Table III. Operative and tumor-related factors according to CRP/Alb ratio on POD14.

\begin{tabular}{|c|c|c|c|}
\hline \multirow[t]{2}{*}{ Variables } & \multicolumn{2}{|c|}{$\mathrm{CRP} / \mathrm{Alb}$ at POD 14} & \multirow[t]{2}{*}{$p$-Value } \\
\hline & $\begin{array}{c}\text { Low }(\leq 0.30) \\
n=40\end{array}$ & $\begin{array}{c}\text { High }(>0.30) \\
n=40\end{array}$ & \\
\hline \multicolumn{4}{|l|}{ Operative factors } \\
\hline Resection type (minor/major) & $13 / 27$ & $6 / 34$ & 0.0813 \\
\hline Blood loss (ml) & $425(5-1974)$ & $635(29-2605)$ & 0.0084 \\
\hline Operative time (min) & $410(221-610)$ & $452(259-799)$ & 0.0433 \\
\hline Biliary resection (yes/no) & $0 / 40$ & $9 / 31$ & 0.0011 \\
\hline Complication $\geq \mathrm{CD}$ IIIa (yes/no) & $5 / 35$ & $14 / 26$ & 0.0163 \\
\hline \multicolumn{4}{|l|}{ Tumor-related factors } \\
\hline Tumor size (mm) & $32(10-120)$ & $35(2-90)$ & 0.4677 \\
\hline Tumor number (single/multiple) & $34 / 6$ & $34 / 6$ & 1.0000 \\
\hline Vascular invasion (yes/no) & $20 / 20$ & $18 / 20$ & 0.6542 \\
\hline pN stage $(\mathrm{X} / 0 / 1)$ & $15 / 11 / 4$ & $16 / 11 / 2$ & 0.707 \\
\hline pStage (I/II/III/IV) & $2 / 6 / 12 / 9$ & $0 / 13 / 11 / 5$ & 0.140 \\
\hline
\end{tabular}

CD: Clavien-Dindo.

$p$-values on Cox-proportional hazard analysis were 0.420 , $0.122,0.543,0.054,0.008,0.069$, and 0.543 preoperatively and on POD1, POD3, POD7, POD14, POM1, and POM3, respectively (Table I). The only statistically significant association was between POD14 and OS. OS curves according to high and low CRP/Alb are shown in Figure 1.

Clinicopathological factors associated with high CRP/Alb ratio on POD 14. Next, we compared selected clinicopathological factors between the high and low CRP/Alb groups on
POD14 (Table II). A high CRP/Alb ratio on POD14 was significantly associated with the clinical characteristics of male sex $(p=0.0025)$, low serum albumin $(p=0.0182)$, and high CRP $(p=0.0017)$. No tumor-related factors were significantly associated with OS (Table III). As to operative factors, a high CRP/Alb ratio on POD14 was significantly associated with longer operation time $(p=0.0433)$, more intraoperative bleeding $(p=0.0084)$, higher morbidity rate $(p=0.0163)$, and higher rate of biliary resection $(p=0.0011)$ (Table III). To determine which clinicopathological factors 
Table IV. Relationships between clinicopathological factors and CRP/Alb ratio on POD 14.

\begin{tabular}{|c|c|c|c|c|c|}
\hline \multirow[t]{2}{*}{ Factors } & \multicolumn{2}{|c|}{ Univariate analysis } & \multicolumn{2}{|c|}{ Multivariate analysis } & \multirow[b]{2}{*}{$p$-Value } \\
\hline & Odds ratio & $p$-Value & Odds ratio & $95 \% \mathrm{CI}$ & \\
\hline Age $\geq 65$ & 2.71 & 0.035 & 4.34 & $1.46-14.63$ & 0.0076 \\
\hline Gender (male) & 4.21 & 0.003 & 5.89 & $2.03-19.43$ & 0.0008 \\
\hline HBs-Ag (positive) & 3.35 & 0.128 & & & $\mathrm{~ns}$ \\
\hline HCV-Ab (positive) & 1.42 & 0.556 & & & $\mathrm{~ns}$ \\
\hline ICG R15 $\geq 10 \%$ & 2.02 & 0.143 & & & ns \\
\hline $\mathrm{CEA} \geq 3.4$ & 2.40 & 0.082 & & & ns \\
\hline CA19-9 $\geq 37$ & 1.10 & 0.822 & & & $\mathrm{~ns}$ \\
\hline Blood loss $\geq 525 \mathrm{ml}$ & 2.81 & 0.024 & & & ns \\
\hline Operative time $\geq 445 \mathrm{~min}$ & 2.50 & 0.043 & & & ns \\
\hline Multiple tumors $\geq 2$ & 1.00 & 1.00 & & & ns \\
\hline Tumor size $\geq 50 \mathrm{~mm}$ & 1.44 & 0.458 & & & ns \\
\hline $\mathrm{pN}(+)$ & 1.07 & 0.929 & & & ns \\
\hline Vascular invasion (+) & 1.22 & 0.654 & & & ns \\
\hline Complication $(+) \geq$ CD IIIa & 3.77 & 0.016 & 8.45 & $2.14-43.44$ & 0.0016 \\
\hline
\end{tabular}

CA19-9: Carbohydrate antigen 19-9; CEA: carcinoembryonic antigen; HBs-Ag: hepatitis B surface antigen; HCV-Ab: hepatitis C virus antibody.

Table V. Prognostic factors for OS.

\begin{tabular}{|c|c|c|c|c|c|}
\hline \multirow[t]{2}{*}{ Factors } & \multicolumn{2}{|c|}{ Univariate } & \multicolumn{2}{|c|}{ Multivariate analysis } & \multirow[b]{2}{*}{$p$-Value } \\
\hline & HR & $p$-Value & HR & $95 \% \mathrm{CI}$ & \\
\hline Age $\geq 65$ & 1.25 & 0.549 & & & ns \\
\hline Gender (male) & 0.84 & 0.640 & & & ns \\
\hline HBs-Ag (positive) & 0.57 & 0.320 & & & ns \\
\hline $\mathrm{HCV}-\mathrm{Ab}$ (positive) & 1.35 & 0.559 & & & ns \\
\hline ICG R $15 \geq 10 \%$ & 1.82 & 0.112 & & & ns \\
\hline $\mathrm{CEA} \geq 3.4$ & 1.45 & 0.391 & & & ns \\
\hline CA19-9 $\geq 37$ & 1.34 & 0.420 & & & ns \\
\hline Blood loss $\geq 525 \mathrm{ml}$ & 1.34 & 0.425 & & & ns \\
\hline Operative time $\geq 445 \mathrm{~min}$ & 1.33 & 0.425 & & & ns \\
\hline Multiple tumors $\geq 2$ & 1.22 & 0.675 & & & ns \\
\hline Tumor size $\geq 50 \mathrm{~mm}$ & 0.95 & 0.901 & & & ns \\
\hline $\mathrm{pN}(+)$ & 3.71 & 0.008 & 4.53 & $1.71-10.68$ & 0.004 \\
\hline Vascular invasion (+) & 1.44 & 0.313 & 2.91 & $1.16-6.03$ & 0.020 \\
\hline Complication $(+) \geq$ CD IIIa & 2.86 & 0.010 & 2.91 & $1.28-6.38$ & 0.012 \\
\hline CRP/Alb POD $14>0.28$ & 2.58 & 0.001 & 2.62 & $1.21-5.88$ & 0.014 \\
\hline
\end{tabular}

CA19-9: Carbohydrate antigen; CEA: carcinoembryonic antigen; 19-9HBs-Ag: hepatitis B surface antigen; HCV-Ab: hepatitis C virus antibody.

were independently associated with the $\mathrm{CRP} / \mathrm{Alb}$ ratio at POD14, selected clinicopathological factors were divided into two groups according to their median values and subjected to logistic regression analysis (Table IV). In this multivariate analysis, age $(\geq 65)$ [odds ratio $(\mathrm{OR})=4.34, p=0.0076$ ], male sex $(\mathrm{OR}=5.89, p=0.0008)$, and the presence of complications $(\mathrm{OR}=8.45, p=0.0016)$ were independently associated with $\mathrm{CRP} / \mathrm{Alb}$ ratio on POD14. Finally, to assess whether CRP/Alb ratio on POD14 more accurately predicts ICC outcomes than other clinicopathological factors, Cox proportional hazard analysis for OS was performed (Table V). Pathological lymph node metastasis $(p=0.004)$, pathological vascular invasion $(p=0.020)$, presence of complications $(p=0.012)$ and high $\mathrm{CRP} / \mathrm{Alb}$ ratio at POD $14(p=0.014)$ were thus identified as independent prognostic factors for poor OS.

\section{Discussion}

To the best of our knowledge, this is the first study to implicate postoperative and perioperative inflammatory score 
and nutrition scores in the prognosis of patients with ICC who have undergone curative resections. Although surgery is the most effective medical treatment for solid tumors, surgical resection is invasive. In particular, resection of ICC sometimes requires major hepatectomy and can result in serious complications such as liver failure or bile leakage (14). Furthermore, comorbidities, such as hypertension and diabetes mellitus are becoming common in older patients undergoing invasive surgery. Although the safety of modern surgical procedures has improved, the risks of morbidity and mortality remain high in these patients. In past reports, the risk of postoperative complications has been estimated by the Physiological and Operative Severity Score for the Enumeration Mortality and Morbidity (POSSUM) and the Estimation of Physiology Ability and Surgical Stress (E-PASS) scoring system, both of which are based on preoperative factors $(23,24)$. On the other hand, biological reactions by surgical stress differ between patients. Experienced gastroenterological surgeons know that patients with perioperative high inflammation and perioperative low nutritional markers are likely to have poor postoperative outcomes, these being indicators of a patient's probable response to surgical stress.

In this study, we assessed the relationship between perioperative $\mathrm{CRP} / \mathrm{Alb}$ ratio and clinical outcomes of patients with ICC. Of the CRP/Alb ratios at different perioperative points, the ratio on POD 14 was significantly associated with prognosis. We believe that one of the main reasons for this finding is that postoperative complications are associated with an increase in serum CRP and a decrease in serum Alb concentration. In fact, the presence of complications was found to be independently associated with a high CRP/Alb ratio on POD14 (Table III). The prognostic value of the $\mathrm{CRP} / \mathrm{Alb}$ ratio on POD14 has also been demonstrated in patients with pancreatic ductal adenocarcinoma; specifically, a high ratio was significantly associated with poor OS and relapse-free survival (25). In the present study, the $\mathrm{CRP} / \mathrm{Alb}$ on POD14 was also associated with the volume of intraoperative blood loss and the duration of the surgical procedure, as well as with older age, which is subsequently linked with severe surgical stress. According to Watt et al. (16), the mGPS on POD 3 is associated with OS and major infectious complications in patients with colorectal cancer who underwent curative resection. However, they investigated the mGPS at only two points, namely on POD 3 and POD 4.

So what is the clinical usefulness of the CRP/Alb ratio on POD 14? We believe that it would assist the postoperative management. For example, patients with high CRP/Alb ratios on POD14 may be good candidates for adjuvant chemotherapy. So far, there is no evidence for the clinical effectiveness of adjuvant chemotherapy after resection of bile duct cancer. Ebata et al. have reported that the probability of survival after resection of bile duct cancer did not differ significantly between patients who received gemcitabine adjuvant chemotherapy and those who were simply observed (26).

The current study had several limitations. First, it was retrospective study and included relatively few patients with ICC attending a single institution. Second, several factors that can affect perioperative inflammation-based and nutritional scores such as medications and meals varied between patients. Thus, a large-scale prospective study is required to confirm our findings.

In conclusion, the CRP/Alb ratio on POD14 is an independent prognostic factor in patients with ICC who have undergone curative resections.

\section{Conflicts of Interest}

The Authors declare that they have no conflicts of interest regarding this study.

\section{Authors' Contributions}

YN and YY participated in the conception, design, analysis of this study and drafted the manuscript. KA, TM, RI, TY, NU, TY, SN, $\mathrm{HO}$ and $\mathrm{KI}$ participated in the statistical analysis and revised it. TA and $\mathrm{HB}$ conceived of the study and participated in its design and coordination and helped to draft the manuscript. All authors read and approved the final manuscript.

\section{References}

1 Khan SA, Taylor-Robinson SD, Toledano MB, Beck A, Elliott P and Thomas HC: Changing international trends in mortality rates for liver, biliary and pancreatic tumours. J Hepatol 37(6): 806813, 2002. PMID: 12445422

2 Shaib YH, Davila JA, McGlynn K and El-Serag HB: Rising incidence of intrahepatic cholangiocarcinoma in the United States: a true increase? J Hepatol 40(3): 472-427, 2004. PMID: 15123362. DOI: 10.1016/j.jhep.2003.11.030

3 Yoon H, Min JK, Lee JW, Kim DG and Hong HJ: Acquisition of chemoresistance in intrahepatic cholangiocarcinoma cells by activation of AKT and extracellular signal-regulated kinase (ERK)1/2. Biochem Biophys Res Commun 405(3): 333-337, 2011. PMID: 21130731. DOI: 10.1016/j.bbrc.2010.11.130

4 de Jong MC, Nathan H, Sotiropoulos GC, Paul A, Alexandrescu S, Marques H, Pulitano C, Barroso E, Clary BM, Aldrighetti L, Ferrone CR, Zhu AX, Bauer TW, Walters DM, Gamblin TC, Nguyen KT, Turley R, Popescu I, Hubert C, Meyer S, Schulick RD, Choti MA, Gigot JF, Mentha G and Pawlik TM: Intrahepatic cholangiocarcinoma: an international multiinstitutional analysis of prognostic factors and lymph node assessment. J Clin Oncol 29(23): 3140-3145, 2011. PMID: 21730269. DOI: $10.1200 / J C O .2011 .35 .6519$

5 Ohtsuka M, Ito H, Kimura F, Shimizu H, Togawa A, Yoshidome $\mathrm{H}$ and Miyazaki M: Results of surgical treatment for intrahepatic cholangiocarcinoma and clinicopathological factors influencing survival. Br J Surg 89(12): 1525-1531, 2002. PMID: 12445060. DOI: $10.1046 / \mathrm{j} .1365-2168.2002 .02268 . x$ 
6 Hanahan D and Weinberg RA: Hallmarks of cancer: The next generation. Cell 144(5): 646-674, 2011. PMID: 21376230. DOI: 10.1016/j.cell.2011.02.013

7 Mantovani A, Allavena P, Sica A and Balkwill F: Cancer-related inflammation. Nature 454(7203): 436-444, 2008. PMID: 18650914. DOI: $10.1038 /$ nature07205

8 Lin G, Liu Y, Li S, Mao Y, Wang J, Shuang Z, Chen J and Li S: Elevated neutrophil-to-lymphocyte ratio is an independent poor prognostic factor in patients with intrahepatic cholangiocarcinoma. Oncotarget 7(32): 50963-50971, 2016. PMID: 26918355. DOI: 10.18632 /oncotarget.7680

9 Chen Q, Dai Z, Yin D, Yang LX, Wang Z, Xiao YS, Fan J and Zhou J: Negative impact of preoperative platelet-lymphocyte ratio on outcome after hepatic resection for intrahepatic cholangiocarcinoma. Medicine 94(13): e574, 2015. PMID: 25837750. DOI: 10.1097/MD.0000000000000574

10 Okuno M, Ebata T, Yokoyama Y, Igami T, Sugawara G, Mizuno T, Yamaguchi J and Nagino M: Evaluation of inflammation-based prognostic scores in patients undergoing hepatobiliary resection for perihilar cholangiocarcinoma. J Gastroenterol 51(2): 153-161, 2016. PMID: 26187429. DOI: 10.1007/s00535-015-1103-y

11 Fairclough E, Cairns E, Hamilton J and Kelly C: Evaluation of a modified early warning system for acute medical admissions and comparison with $\mathrm{C}$-reactive protein/albumin ratio as a predictor of patient outcome. Clin Med (Lond) 9(1): 30-33, 2009. PMID: 19271597.

12 Ranzani OT, Zampieri FG, Forte DN, Azevedo LC and Park M: C-reactive protein/albumin ratio predicts 90-day mortality of septic patients. PLoS One 8(3): e59321, 2013. PMID: 23555017. DOI: 10.1371 journal.pone.0059321

13 Kinoshita A, Onoda H, Imai N, Iwaku A, Oishi M, Tanaka K, Fushiya N, Koike K, Nishino H and Matsushima M: The Creactive protein/albumin ratio, a novel inflammation-based prognostic score, predicts outcomes in patients with hepatocellular carcinoma. Ann Surg Oncol 22(3): 803-810, 2015. PMID: 25190127. DOI: 10.1245/s10434-014-4048-0

14 Miyata T, Yamashita YI, Yamao T, Umezaki N, Tsukamoto M, Kitano Y, Yamamura K, Arima K, Kaida T, Nakagawa S, Imai K, Hashimoto D, Chikamoto A, Ishiko T and Baba H: Prognostic impacts of postoperative complications in patients with intrahepatic cholangiocarcinoma after curative operations. Int J Clin Oncol 22(3): 526-532, 2017. PMID: 28205002. DOI: 10.1007/s10147-017-1099-9

15 Khuri SF, Henderson WG, DePalma RG, Mosca C, Healey NA, Kumbhani DJ and Participants in the VA National Surgical Quality Improvement Program: Determinants of long-term survival after major surgery and the adverse effect of postoperative complications. Ann Surg 242(3): 326-341, 2005. PMID: 16135919.

16 Watt DG, McSorley ST, Park JH, Horgan PG and McMillan DC: A postoperative systemic inflammation score predicts short- and long-term outcomes in patients undergoing surgery for colorectal cancer. Ann Surg Oncol 24(4): 1100-1109, 2017. PMID: 27822634. DOI: 10.1245/s10434-016-5659-4

17 Yamashita Y, Taketomi A, Morita K, Fukuhara T, Ueda S, Sanefuji K, Iguchi T, Kayashima H, Sugimachi K and Maehara $\mathrm{Y}$ : The impact of surgical treatment and poor prognostic factors for patients with intrahepatic cholangiocarcinoma: retrospective analysis of 60 patients. Anticancer Res 28(4C): 2353-2359, 2008. PMID: 18751418 .
18 Yamashita YI, Wang H, Kurihara T, Tsujita E, Nishie A, Imai K, Hashimoto D, Chikamoto A, Aishima S and Baba H: Clinical significances of preoperative classification of intrahepatic cholangiocarcinoma: different characteristics of perihilar vs. peripheral ICC. Anticancer Res 36(12): 6563-6569, 2016. PMID: 27919984. DOI: 10.21873 /anticanres.11260

19 Miyata T, Yamashita YI, Yamao T, Umezaki N, Tsukamoto M, Kitano Y, Yamamura K, Arima K, Kaida T, Nakagawa S, Imai K, Hashimoto D, Chikamoto A, Ishiko T and Baba H: Clinical benefits of lymph node dissection in intrahepatic cholangiocarcinoma: a retrospective single-institution study. Anticancer Res 37(5): 26732677, 2017. PMID: 28476843. DOI: 10.21873/anticanres.11615

20 Adachi T, Eguchi S, Beppu T, Ueno S, Shiraishi M, Okuda K, Yamashita Y, Kondo K, Nanashima A, Ohta M, Takami Y, Noritomi T, Kitahara K and Fujioka H: Prognostic impact of preoperative lymph node enlargement in intrahepatic cholangiocarcinoma: A multi-institutional study by the Kyushu study group of liver surgery. Ann Surg Oncol 22(7): 2269-2278, 2015. PMID: 25582737. DOI: 10.1245/s10434-014-4239-8

21 Dindo D, Demartines N and Clavien PA: Classification of surgical complications: a new proposal with evaluation in a cohort of 6336 patients and results of a survey. Ann Surg 240(2): 205-213, 2004. PMID: 15273542.

22 Edge S, Byrd DR, Compton CC, Fritz AG, Greene FL and Trotti A: AJCC Cancer Staging Manual (7th ed). New York, NY, Springer. 2010.

23 Copeland GP, Jones D and Walters M: POSSUM: a scoring system for surgical audit. Br J Surg 78(3): 355-360, 1991. PMID: 2021856.

24 Haga Y, Ikei S and Ogawa M: Estimation of physiologic ability and surgical stress (E-PASS) as a new prediction scoring system for postoperative morbidity and mortality following elective gastrointestinal surgery. Surg Today 29(3): 219-225, 1999. PMID: 10192731. DOI: 10.1007/BF02483010

25 Arima K, Yamashita YI, Hashimoto D, Nakagawa S, Umezaki N, Yamao T, Tsukamoto M, Kitano Y, Yamamura K, Miyata T, Okabe $\mathrm{H}$, Ishimoto $\mathrm{T}$, Imai $\mathrm{K}$, Chikamoto $\mathrm{A}$ and Baba $\mathrm{H}$ : Clinical usefulness of postoperative C-reactive protein/albumin ratio in pancreatic ductal adenocarcinoma. Am J Surg 216(1): 111-115, 2017. PMID: 28859917. DOI: 10.1016/j.amjsurg.2017.08.016

26 Ebata T, Hirano S, Konishi M, Uesaka K, Tsuchiya Y, Ohtsuka M, Kaneoka Y, Yamamoto M, Ambo Y, Shimizu Y, Ozawa F, Fukutomi A, Ando M, Nimura Y and Nagino M; Bile Duct Cancer Adjuvant Trial (BCAT) Study Group: Randomized clinical trial of adjuvant gemcitabine chemotherapy versus observation in resected bile duct cancer. Br J Surg 105(3): 192202, 2018. PMID: 29405274. DOI: 10.1002/bjs.10776
Received January 31, 2019

Revised March 30, 2019

Accepted April 9, 2019 